\title{
Body image, visual working memory and visual mental imagery
}

Stephen Darling, Clare Uytman, Richard J Allen, Jelena Havelka, David G Pearson

Body dissatisfaction (BD) is a highly prevalent feature amongst females in society, with the majority of individuals regarding themselves to be overweight compared to their personal ideal, and very few self-describing as underweight. To date, explanations of this dramatic pattern have centred on extrinsic social and media factors, or intrinsic factors connected to individuals' knowledge and belief structures regarding eating and body shape, with little research examining links between $\mathrm{BD}$ and basic cognitive mechanisms. This paper reports a correlational study in which visual and executive cognitive processes that could potentially impact on BD were assessed. Visual memory span and self-rated visual imagery were found to be predictive of $\mathrm{BD}$, alongside a measure of inhibition derived from the Stroop task. In contrast, spatial memory and global precedence were not related to BD.

Results are interpreted with reference to the influential multi-component model of working memory. 
${ }^{1}$ Division of Psychology and Sociology, Queen Margaret University, Edinburgh, UK

* Corresponding author

8

9

10

11

12

13

14

15

16

17

18

19

22 Correspondence concerning this article should be addressed to Stephen Darling, Division of

23 Psychology and Sociology, Queen Margaret University, Edinburgh EH21 6UU, UK. 
Abstract

Body dissatisfaction (BD) is a highly prevalent feature amongst females in society, with the

28 majority of individuals regarding themselves to be overweight compared to their personal ideal, and

29 very few self-describing as underweight. To date, explanations of this dramatic pattern have centred on

30 extrinsic social and media factors, or intrinsic factors connected to individuals' knowledge and belief

31 structures regarding eating and body shape, with little research examining links between $\mathrm{BD}$ and basic

32 cognitive mechanisms. This paper reports a correlational study in which visual and executive cognitive

33 processes that could potentially impact on BD were assessed. Visual memory span and self-rated visual

34 imagery were found to be predictive of $\mathrm{BD}$, alongside a measure of inhibition derived from the Stroop

35 task. In contrast, spatial memory and global precedence were not related to BD. Results are interpreted

36 with reference to the influential multi-component model of working memory. 
Body image is a general term attached to a complex web of constructs by which individuals

40 relate to their own bodies, and in particular to their appearance. In western societies the issue of weight

41 (thinness) has been of particular significance for body image, as societal ideals have tended to

42 emphasize unattainable degrees of thinness, especially in females (Tiggemann, 2006). These unrealistic

43 ideals may underlie what has been termed a 'normative discontent' (Rodin, Silberstein \& Striegel-

44 Moore, 1984). There is little doubt that body dissatisfaction (BD) contributes greatly to levels of

45 distress in the general population (Ohring, Graber, \& Brooks-Gunn, 2002; Thompson, 1999), and is

46 related to onset of pathological eating disorders (Attie \& Brooks-Gunn, 1989; Killen et al., 1996).

47 Furthermore, although females and males both show evidence of BD at roughly equivalent prevalence

48 (e.g. Furnham \& Calnan, 1998; Furnham, Badmin \& Sneade, 2002), the nature of dissatisfaction is

49 qualitatively different between the sexes: the vast majority of females expressing BD express a

50 preference for a slimmer figure, whilst for males the distribution is roughly symmetrical, with many

51 wishing to be bulkier (Furnham \& Calnan, 1998; Furnham et al, 2002; Drewnowski \& Yee, 1987).

An increased knowledge of the predictors and correlates of BD is necessary in order to further

53 understand this issue. A good deal is already understood about such predictors: factors such as

54 environmental social pressure to be thin, weight-related teasing, internalization of a thin-ideal, lack of

55 social support, individual differences in body mass and peer pressure have all been identified as

56 specific predictors of body dissatisfaction in adolescent girls (Stice \& Whitenton, 2002, Presnell,

57 Bearman and Stice, 2004).

Although extrinsic social forces such as those detailed above undoubtedly shape body

59 dissatisfaction, it is important to probe intrinsic factors that may be related to negative body image

60 evaluation too. There is, for example, clear evidence for the existence of a relationship between

61 negative affect and body dissatisfaction (Presnell, et al 2004; Konstanski \& Gullone, 2003), though 
62 longitudinal research suggests that early BD predicts later depression rather than vice versa (Stice,

63 Hayward, Cameron, Killen \& Taylor, 2000; Paxton, Neumark-Sztainer, Hannan, Eisenberg, 2006).

64 Other intrinsic factors linked to $\mathrm{BD}$ include dispositional factors, personality factors and attachment

65 (Abbate-Daga, Gramaglia, Amianto, Marzolo \& Fassino, 2010), alongside biological factors such as

66 adiposity (e.g. Stice \& Whitenton, 2002).

Information-processing factors also have the potential to impact on BD. In the last twenty years an understanding of the constraints imposed by cognitive architecture has had a profound impact on understanding of social processing (see, e.g. Fazio, Jackson, Dunton \& Williams, 1995; Greenwald, Nosek \& Banaji, 2003). In a similar vein, a body of research has emerged focusing on information

71 processing and $\mathrm{BD}$ (for a review see Lee \& Shafran, 2004). This research has broadly shown that

72 individuals with eating disorders attend differentially to information pertaining to shape, weight and

73 eating (e.g. Vitousek \& Hollon, 1990) and body components (Freeman et al., 1991); Fairburn, Shafran

74 \& Cooper, 1999). There is also a range of evidence from modifications of the Stroop task (Stroop,

75 1935) where participants are required to report the colour of ink in which a word is printed. In the

76 emotional variant of the Stroop task, neutral and emotionally charged words are presented to

77 participants who are then asked to identify the print colour of those words. It has been demonstrated

78 that unpleasant words (e.g., fear) produce greater slowing down of reaction times than neutral words

79 (e.g., leaf). This pattern has also been observed in clinical populations. For example, words related to

80 specific phobias (i.e. arachnophobia) result in slower response times when presented to individuals

81 with those phobias (for a review see Williams, Mathews, \& MacLeod, 1996), while alcohol related

82 words produce slower response times in alcoholics (e.g. Lusher, Chandler, \& Ball, 2004). In relation to

83 the present study, participants with a high 'drive for thinness' demonstrated greater impairment in ink

84 colour naming latency when the ostensibly irrelevant text was composed of food words than control

85 words (Perpiñá, Hemsley, Treasure \& De Silva, 1993); participants who were high in dietary restraint 
86 showed a similar pattern towards food and body shape words (Green \& Rogers, 1993). There is also a

87 relationship between the retention of body-related items in memory and BD (Baker, Williamson \&

88 Sylve, 1995;). Hargreaves and Tiggeman (2002) reported that declines in girls' body satisfaction were

89 related to the strength of cognitive schemata underlying appearance at the start of the research period,

90 and that this was distinguishable from self-esteem. The picture emerging from this literature is that of a

91 relationship between biases in cognition and information processing centred around concepts related to

92 the body and food, and increased body dissatisfaction or proneness to eating disorders.

The cognitive factors described above, however, are intrinsically linked to the conceptual

understanding of bodies and food, and are likely to reflect the content of semantic memory related to

95 food and body related self-image. There are a further set of cognitive functions that may influence

96 body dissatisfaction, these being more abstract cognitive processes that likely influence a range of

97 behaviours such as, for example, planning and inhibition. There is, for example, evidence that poor

98 inhibition, as assessed on the colour-word Stroop task, is associated with increased obesity, though not

99 with anorexia nervosa (Fagundo et al., 2012), alongside other evidence of links between obesity and

100 impaired executive functions such as inhibition, flexibility and planning (Boeka \& Lokken, 2008,

101 Cserjési, Luminet, Poncelet \& Lénárd, 2009, Cserjési, Molnár, Luminet \& Lénárd, 2007, Gunstad et

102 al., 2007, Nederkoorn, Braet, Van Eijs, Tanghe \& Jansen, 2006a, Nederkoorn, Smulders, Havermans,

103 Roefs \& Jansen, 2006b). There are also relationships between eating disorders and executive functions

104 (for reviews see: Aspen, Darcy \& Lock, 2013; Dobson \& Dozois, 2004; Lena, Fiocco \& Leyenaar,

105 2004; Roberts, Tchanturia, Stahl, Southgate \& Treasure, 2007).

106

Our purpose here is to focus in more detail on one particular aspect of cognition that has been

107 largely neglected in understanding body dissatisfaction and related issues: visual cognition. The

108 research reported here starts from a simple and relatively uncontroversial position - that there are (at

109 least) two representative codes capable of sustaining imagery in the cognitive system, one verbal and 
110 one visuospatial in nature (Paivio, 1991). This dual coding approach underlies the highly influential

111 Working Memory approach (Baddeley \& Hitch, 1974; Baddeley, Allen \& Hitch, 2011). In essence the

112 working memory approach posits that parallel temporary verbal and visual information storage systems

113 (known respectively as the phonological loop and visuo-spatial sketch pad) store information in a

114 passive manner, whilst other multimodal systems can perform operations on or form links to that

115 information. One type of multimodal system is the 'central executive' - a label reflecting a set of

116 largely active processes that can manipulate the information held temporarily active in the passive

117 stores, whilst the another, termed the 'episodic buffer' is more automatic in nature and involved in the

118 formation of cohesive and unified 'episodes' (Baddeley, et al., 2011). When looked at from this

119 perspective it seems likely that any representation of body image is likely to contain a visuospatial

120 component alongside a verbal one. There is ample evidence that such visual images can be created and

121 maintained in memory, even in the absence of explicit visual input (e.g. Quinn \& McConnell, 1996;

122 Pearson \& Logie, 2004). Any task where a participant is asked to consider their own body image

123 should invoke, at least to some extent, a visual representation. Hence, visual imagery ability should be

124 linked to body image. One possibility is that the more realistic and veridical a participant's internal

125 model of their own appearance is, the more resistant they should be to bias and distortion of body

126 image driven by social and cultural factors operationalized in semantic memory.

127 Smeets, Klugkist, van Rooden, Anema, and Postma (2009) have postulated a link between

128 visual imagery processes and body image using a variant of the Distance Comparison task (Kosslyn,

129 Ball, \& Reiser, 1978). In their study participants were asked to mentally scan across two specified body

130 widths (e.g., hips and waist) and indicate as quickly as possible which body part was longer or shorter.

131 The results showed females with high BD were significantly less accurate on body width comparisons

132 involving small (less than $9 \mathrm{~cm}$ ) differences, implying that they had a less accurate visual image of

133 their own body in comparison to a group of low BD females. 
135 cannot directly assess a participant's subjective experience of mental imagery. Auchus, Kose and

136 Allen (1993) report a study that, surprisingly, appears to be the only attempt to date to study the

137 relationship between subjective self-reported visual imagery and body imagery. This study subdivided

138 a sample on a post-hoc basis into four groups based on a test where they had to adjust the appearance

139 of an initially distorted image of themselves until they felt it reflected reality. In a control test they

140 adjusted the shape of an object (a tin of soup) in the same way. Participants were assessed to be either

141 non-distorters, distorters of object but not own-body representations, distorters of own-body

142 representations but not objects, and general distorters. Various different measures of visual imagery

143 were taken: overall, no significant group differences were identified.The authors also adopted an

144 extreme groups approach and claimed to observe increased ratings of imagery vividness (using the

145 Vividness of Visual Imagery Questionnaire - VVIQ: Marks, 1973) when a subset of the participants

146 who distorted body image the most was compared against the subset that distorted the least. Such an

147 approach is methodologically problematic when coupled with small sample size, and does not appear

148 to have been replicated in the literature. In order to clarify and consolidate understanding of the

149 relationship of visual imagery and body imagery, the current study adopts a correlational design in

150 which a number of cognitive measures are adopted in order to predict BD in a female sample. We

151 focus specifically on females because the aforementioned evidence that BD has a quite different profile

152 in females and males would imply that separate consideration of body image issues in females and

153 males would be appropriate.

154 The selection of measures was informed by current understandings of visual imagery and visual

155 WM. They included a self-report measure of the vividness of visual imagery (the VVIQ), computerized

156 versions of the Corsi Block task (Corsi, 1972; Milner, 1971) and the Visual Patterns Task (VPT: Della 
157 Sala, Gray, Baddeley \& Wilson, 1997). We also included a measure of global precedence, and a 158 standard ink-naming colour word version of the Stroop task.

159 The VVIQ allows participants to self-report their use of visual imagery. There is evidence that

160 it does index visual working memory, but it clearly also implicates elements of long-term memory

161 (Baddeley \& Andrade, 2000). Imagery tasks also tend to implicate executive resources (Logie, 1995,

162 2003; Pearson, Logie \& Green, 1996; Rudkin, Pearson \& Logie, 2007; Quinn \& Ralston, 1986).

163 Consequently, in order to incorporate more specific measures of visuospatial working memory

164 processing, two direct measures of visual and spatial memory span were used. There is evidence (Della

165 Sala, Gray, Baddeley, Allamano \& Wilson, 1999) that the VPT and Corsi tasks measure qualitatively

166 different visual and spatial sub-processes of working memory, and hence together they provide the

167 opportunity to understand relationships with BD in a more sophisticated way. Global precedence is a

168 calculated score reflecting the degree to which a person tends to view visual arrays in a global or a

169 local manner. Global processors may remember faces better (Darling, Martin, Hellman \& Memon,

1702009 ) and process information about faces in a more configural, holistic fashion (Martin \& Macrae,

171 2010). It is included here to see if this preference for broad configural information over small-scale

172 local detail is related to the tendency to misrepresent ones' own body. Somewhat relatedly, Feusner,

173 Moller, Altstein, Sugar, Bookheimer, Yoon and Hembacher (2010) observed a predisposition to local

174 processing on a face inversion task amongst a sample of individuals with Body Dysmorphic Disorder 175 (BDD).

176 As well as direct assessments of visual memory, we also included a measure of resistance to

177 inhibition by irrelevant information - the classic Stroop task (Stroop, 1935). This task was included as

178 an estimate of executive functioning, in particular indexing attention and inhibition (for a review see

179 MacLeod, 1991), two of the so-called executive functions (e.g. Baddeley, 1996; Miyake, Freidman,

180 Emerson, Witzki, Howerter \& Wager, 2000). Although we acknowledge that executive function is a 
181 concept encompassing a diversity of elements, the Stroop task provides a simple and straightforward 182 method of assessing at least some of these components. The classic colour-word interference version of

183 the Stroop task used in this experiment measures the interfering effect of a colour word on the

184 reporting of the colour of ink in which word is presented. Hence the word 'RED' might be printed in

185 blue ink, and the participant would be required to report the ink colour as 'blue'. Successful completion

186 of the task requires the participant to inhibit the prepotent response (reading out the colour word 'red').

187 This task, therefore, does not tap inhibition or orientation to particular concepts, but indexes general

188 inhibitory functioning. Although poor inhibition control has been implicated in eating disorders and

189 obesity, as discussed above, and although the ability to suppress undesired thoughts may be useful in

190 mitigating against body dissatisfaction itself, there is comparatively little evidence linking body

191 dissatisfaction to Stroop performance. Hence it is an open question whether Stroop performance will

192 relate to body dissatisfaction.

193 In summary, this report presents a study in which we sought to assess the relative importance of

194 various visual imagery, visual memory and executive cognitive factors, with no direct link to eating

195 behavior, in predicting BD in a sample of females. Age and obesity, assessed using Body Mass Index

196 (BMI $)^{1}$, were also assessed as predictors, so that an assessment of the role of cognitive factors could be

197 made independent of age and BMI. To do this we adopted two broadly accepted measures of body

198 dissatisfaction, a verbal questionnaire (a short form of the Body Shape Questionnaire, the BSQ-16A:

199 Evans \& Dolan, 1993) and a comparison of ideal and current visual-analogue body shape scales.

Method

201 Participants

202 The sample comprised 111 female participants who were either students or staff of Queen

203 Margaret University, or members of the broader Edinburgh community. Participants took part either

${ }^{1} \mathrm{BMI}=$ height $/(\text { mass })^{2}$ 
204 voluntarily or in return for course credit. Self-reported colour blindness was an exclusion criterion, and 205 participants were required to have normal or corrected-to-normal vision. Mean age of participants was 20626.5 years $(\mathrm{SD}=10.2)$. All participants expressed informed consent in writing and the research was 207 approved at the Psychology and Sociology Subject area level by the Queen Margaret University 208 Research Ethics Panel.

209 Instruments Body Shape Questionnaire

The 16-item Body Shape Questionnaire (BSQ-16A: Evans \& Dolan, 1993) is a self-report scale 212 where participants rate the frequency of various body shape-related feelings over a period of four 213 weeks (e.g. 'Has worry about your shape made you diet?'). It is a shortened form of the original BSQ-

21434 (Cooper, Taylor, Cooper \& Fairburn, 1987), which has good psychometric properties (Evans \& 215 Dolan, 1993). Ratings are expressed as a 6-point Likert scale ranging from 1 (Never) to 6 (Always), 216 and the reported score is the total of these ratings: thus BSQ-16A scores have a potential range from 16 217 to 96. scales (based on Stunkard, Sørensen \& Schulsinger, 1983). Dissatisfaction is assessed by subtraction of

221 the ideal from the current rating, quantifying the discrepancy between current and ideal body 222 representation. This method is regarded as an effective measure of BD (e.g. Bulik, Wade, Heath, 223 Martin, Stunkard \& Eaves, 2001; Mciza, Goedecke, Steyn, Charlton, Puoane, Meltzer, Levitt \& 224 Lambert, 2005) and correlates with BSQ (Mciza et al, 2005). A higher value indicates a greater degree 225 of dissatisfaction, with the current status being regarded as larger than the desired. 
Vividness of Visual Imagery Questionnaire

An unaltered version of Marks' (1973) Vividness of Visual Imagery Questionnaire (VVIQ) was used. Participants are asked to remember 4 scenarios (e.g. 'think of some relative or friend whom you frequently see ... and consider carefully the picture that comes before your mind's eye') and for each of these they are asked to attend to 4 key visual features (e.g. 'The precise carriage, length of step, etc. in walking'). For each of these 16 features, participants give a rating of the intensity of the visual image, on a scale from 1 ('perfectly clear and as vivid as normal vision' to 5 ('no image at all - you only 'know' that you are thinking of an object'). Participants carry out the task twice, first with eyes open, and then with eyes closed. The VVIQ score is simply the total score across all 32 items: note that the scoring is counterintuitive: a lower score on the VVIQ represents more vivid imagery. Visual Patterns Task The Visual Patterns Task (VPT - Della Sala, et al., 1997; Della Sala, et al., 1999) is a task designed to assess memory for static visual patterns without the need for participants to recall sequential information. It is a span task, in which the task difficulty is gradually increased until participants fail to recall a pattern. The current study did not use the original VPT, instead an electronic implementation of the VPT was presented.

\section{Corsi Blocks Task}

The Corsi Blocks task (CBT - Corsi, 1972; Milner, 1971) requires participants to recall a sequence of locations tapped out sequentially on a board with a number of blocks randomly distributed across its surface, and is considered to be a task that indexes spatial sequential memory as distinct from visual pattern memory (Della Sala et al, 1999). In this study a computerized version of the task was used.

\section{Global Precedence}

Global Precedence was measured by adopting a shortened version of the global/local 
250 processing task adopted by Darling et al (2009) and based on the work of Navon (1977). It provides an

251 assessment of the degree to which irrelevant global information is automatically processed compared to 252 irrelevant local information, controlling for general distractibility: the higher the outcome, the more 253 'global' people are in their outlook, at least to the extent of processing irrelevant global information. $254 \quad$ Stroop Interference

255 This test was closely modeled after the ink-colour naming word interference task developed by 256 Stroop (1935), and is described in detail in the procedure section. Naming time in a non-conflict 257 condition was subtracted from naming time in a conflicting condition (where ink colour conflicted with 258 the printed word) to give a Stroop Interference (SI) score. A lower score therefore reflects greater 259 ability to inhibit irrelevant information.

\section{Procedure}

After prviding informed consent, participants had their BMI measured, and then completed the

263 VABD task: they were presented with a page containing two sets of 9 silhouettes of female figures

264 ranging from severely underweight (1) to severely overweight (9) and asked to indicate which figure 265 most closely matched what they currently looked like on the upper set of silhouettes. They then 266 marked, on the lower set, which item represented the shape that they would most like to look like.

267 After this they completed the paper-based BSQ and VVIQ questionnaires. Participants then took part in 268 the Stroop interference task, in which they were shown a sheet containing a $4 \times 6$ grid comprising the 269 letter string 'XXXXX' represented in either blue, green, red, purple, yellow, or orange ink. Participants 270 were asked to name all of the colours and were timed from start to finish to assess the speed with 271 which they could correctly name all 24 . This represented the non-conflicting condition. They were then 272 asked to complete a similar task again, except in this second task, the 'XXXXXX' strings were 
273 replaced by colour names which conflicted with the ink colour that was to be reported (i.e. the word

274 'ORANGE' was depicted in blue ink).

The remaining three tasks were presented on a a standard laptop computer with a 15.7" display

276 (1024 x 768 pixels/319 x $239 \mathrm{~mm})$. First, Global precedence was assessed using a task intended to

277 assess participants' background inclination to process information on a global or a local basis.

278 Participants saw 72 letter displays, in each of which a large letter was comprised of a number of

279 smaller letters (e.g. a letter 'P' was formed from a number of individual letter ' $U$ 's). These displays

280 were modeled on the stimuli used by Navon (1977). Participants were asked to respond by pressing

281 letter keys on the keypad. On 36 trials, the letter display was preceded by an instruction to report the

282 large letter; on the remaining 36 trials participants were told to report the small letter. The instruction

283 used was randomized across trials. For global trials, a local-to-global conflict score was derived by

284 subtracting mean RTs for correct responses for non-conflicting stimuli (where the global and local

285 letters were the same) from correct RTs for conflicting stimuli, yielding a value which increases with

286 the degree to which conflicting local information impaired global processing. For local trials, a global-

287 to-local score was derived in a similar manner reflecting the degree to which conflicting global

288 information impaired local processing. Finally, the global precedence (GP) score was calculated by

289 subtracting the local-to-global conflict score from the global-to-local score. This method yields a

290 global precedence score which takes an increasingly positive value the greater the degree to which

291 global-to-local interference exceeds local-to-global interference.

292 Following the Global Precedence task, participants undertook the Corsi task. This was

293 implemented as a span task, with difficulty increasing until the point that participants were no longer

294 able to successfully reproduce the spatial pattern. Participants saw a set of 9 black outlines of squares

295 (of side 60 pixels $/ 19 \mathrm{~mm}$ ) distributed randomly across a white square in the middle of the screen (side:

$296600 / 190 \mathrm{~mm}$ pixels). A sequence was then traced by randomly highlighting one of these squares at a 
297 time by filling it in a green colour for 500ms. In the first trial, only one item was highlighted. If (on

298 this or any subsequent trial) participants recalled the sequence correctly, the sequence length increased

299 by one item. If an error was made, then a further trial of the same difficulty was presented. Successful

300 recall of this trial enabled the participant to continue at the next level of difficulty, but the procedure

301 stopped after two trials at any difficulty level were erroneously responded to. Participants were

302 allocated a score representing the maximum sequence length that had been correctly remembered

303 during the task: hence a higher score represents a higher capacity for remembering spatial sequences.

304 Finally the visual patterns task was undertaken. Participants were presented with grid arrays

305 comprising a number of individual blank squares of side 60 pixels $(19 \mathrm{~mm})$. Arrays ranged in size from

$3062 \times 1$ cells to a maximum of $6 \times 5$. In each array, $50 \%$ of the squares (at random) were coloured blue.

307 Arrays were visible for a total duration of $250 \mathrm{~ms}$ per visible square (filled or unfilled), meaning larger

308 displays were on screen for longer. Following presentation, a blank grid was shown on the screen.

309 Participants were asked to use a standard mouse to select the squares that had previously been shown in

310 blue. Clicked squares changed colour to indicate that they had been selected. Note that use of the

311 mouse for collecting responses in the visual pattern and Corsi tasks could possibly enlist visuo-spatial

312 skills not engaged in the original versions of these measures.

Results

318 interference score of 193, some 49 standard deviations above the mean of other participants). Two

319 further participants did not contribute data to the global/local precedence task due to electronic data

320 collection failures in that task. Table 1 details summary statistics of the principal body image and 
321 visual imagery measures in the study for all included participants. Correlations between the variables

322 are reported in Table 2. Global precedence showed no correlations with any of the variables except a

323 small relationship with age (i.e. increased local focus with age). Focusing on relationships between the

324 cognitive measures and measures related to body dissatisfaction, VVIQ correlated positively with BSQ

325 and VABD, showing that higher scores (lower imagery) was related to higher body dissatisfaction.

326 VPT correlated negatively with these measures, showing the same pattern - higher scores (higher

327 visual memory performance) related to lower body dissatisfaction, though effect sizes were smaller

328 than for VVIQ correlations. Correlations between Corsi performance and body dissatisfaction scores

329 were not significant. Stroop interference correlated with both BSQ and VABD. There were also

330 notably strong relationships between VABD, Stroop and BMI.

331 To address the fundamental hypotheses of this paper of links between body dissatisfaction and

332 visuospatial working memory, the relative importance of VPT span, Corsi span, global precedence,

333 VVIQ and Stroop interference as predictors of body image was assessed in separate regression

334 analyses upon BSQ and VABD. Age and BMI were included in these analyses as potential covariates

335 of body image. Table 3 reports the results of these analyses. BMI and age were directly entered into the

336 models at Step 1: following this the remaining cognitive predictors (VVIQ, VPT, Corsi, GP and SI)

337 were allowed to enter using a stepwise procedure (probability criteria for entry/removal were $\mathrm{p}<=.05$ /

$338>=.10)$. To avoid undue influences of individual data points on the regression model, participants with

339 Cook's D values exceeding 0.037 (i.e. 4/n) were excluded from each analysis. The resulting models are

340 detailed in Table 3. The models showed that BSQ scores were reliably $(F(5,93)=12.113, \mathrm{p}<0.001$ :

$\left.341 R^{2}=.39\right)$ predicted by a regression model including VVIQ $(t(97)=5.10, \mathrm{p}<.001)$, age $(t(97)=-3.22, p$

$342=.002)$, Stroop interference $(t(97)=2.42, p=.02)$ and VPT $(t(97)=-2.04, \mathrm{p}=.04)$, and that VABD

343 scores were reliably $\left(F(4,102)=14.23, p<0.001: R^{2}=.37\right)$ predicted by a regression model including

$344 \operatorname{BMI}(t(101)=4.73, p<.001)$, VVIQ $(t(101)=3.95, p<.001)$ and VPT $(t(101)=-3.321, p=.001)$. 
Discussion

347 visuospatial and cognitive measures and body dissatisfaction. The relationships identified are between

348 body dissatisfaction and respectively visual mental imagery (vivid imagery is associated with lower

349 dissatisfaction) and visual short-term memory (better memory is associated with lower dissatisfaction).

350 In stark contrast to these patterns, there was no evidence of any link between BD and either spatial-

351 sequential short-term memory or global precedence.

352 These data replicate and considerably extend the results reported by Auchus et al. (1993), who

353 described a similar pattern based on an extreme groups analysis whereby the subset of participants who

354 had the most distorted body image representation reported less rich imagery on the VVIQ, but that

355 these groups did not differ in terms of their visual recall. Unfortunately, the robustness of Auchus et

356 al.'s data is unclear, due both to the low sample size in the extreme groups analyses, and the fact that

357 when an inclusive analysis of the data was carried out there were no differences between body image

358 distorters on either VVIQ or visual memory. The current results therefore substantively strengthen our

359 understanding of the role of both visual imagery and memory in body imagery, firstly by convincingly

360 replicating (with a larger sample and more appropriate analysis, therefore obtaining more satisfactory

361 statistical evidence) the link between self-reported visual imagery (VVIQ) and BD, and secondly by

362 establishing a link between short-term visual retention (VPT) and BD: this latter outcome is entirely

363 novel. In doing so, the role of visual cognitive factors in body dissatisfaction is made explicit:

364 participants with better visual memory and greater self-reported mental imagery are less likely to report

365 being dissatisfied with their appearance. Given these results, it is necessary to ask what the

366 mechanisms may be by which mental imagery and visual memory might influence body image: we

367 address each concept separately. 
Visual mental imagery reflects the conscious internal representation of visual scenes.

369 Consequently it is a very difficult phenomenon to assess. Indeed there has been a historic debate

370 between researchers arguing around its very existence as a separate and characteristic entity. One view

371 holds that imagery is entirely verbal and propositional (Pylyshyn, 1981) and hence functions similarly

372 to linguistic propositional knowledge. An alternative view contends that images depict analogue visual

373 scenes in a direct manner (Kosslyn, 1981), based on pictorial representations. Nonetheless, there is

374 some consensus that the VVIQ indexes a reasonably valid construct (McKelvie, 1995), and in doing so

375 provides subjective evidence in favour of pictorial representations. Why might such a system be related

376 to body dissatisfaction? The answer to such a question awaits future research but one possibility is that

377 an efficient pictorial representational system may serve as a mechanism for storing a consistent and

378 reliable visual self-percept that would be robust against biases imposed from the outside world, for

379 example social and media pressure. In contrast, individuals who have less vivid imagery may find their

380 internal representations can be deflected more easily by external pressure and this can lead to greater

381 inconsistency in comparing self-representation with idealized representations. This interpretation is

382 consistent with the claim made by Smeets et al. that high BD may be associated with less accurate

383 visual imagery for a person's body (Smeets et al., 2009).

Our findings can be related to a growing body of literature that demonstrates an important

385 functional role for mental imagery in clinically-related disorders (see Pearson, Deeprose, Wallace-

386 Hadrill, Burnett Heyes, \& Holmes, 2013). For example, patients with schizophrenia and high-

387 schizotypy controls show significantly greater vividness of imagery than low-schizotypy controls

388 (Oertel et al., 2009). Participants with depression have also been found to be significantly slower at

389 generating visual mental imagery than non-depressed controls (Cocude, Charlot, \& Denis, 1997).

390 Furthermore, selective dual-task interference procedures derived from the Working Memory model

391 (Baddeley, 2000; Baddeley et al, 2011) have been applied to disrupt or reduce clinically-related mental 
392 imagery. Dynamic visual noise is a passive interference procedure known to selectively disrupt visual

393 working memory (McConnell \& Quinn, 2000; Quinn \& McConnell, 2006), and Kemps and Tiggemann

394 (2013) have recently shown that exposure to a hand-held version can significantly reduce naturally

395 occurring food cravings linked to mental imagery. Performance of the demanding visuo-spatial game

396 'Tetris' has also been shown to significantly reduce the occurrence of intrusive mental images for

397 traumatic film material (Holmes, James, Coode-Bate, \& Deeprose, 2009).

398 Some caveats of the current study should be considered. One problem with assessing visual

399 cognition using imagery questionnaires is that the task may not be particularly pure: first, reports are

400 subjective: it is not clear to what extent the self reports are a valid reflection of internal imagery

401 (though it is to be noted that there is evidence that VVIQ scores are affected by manipulations that are

402 known to impact on visuo-spatial working memory: Andrade, Kavanagh \& Baddeley, 1997; Baddeley

$403 \&$ Andrade, 2000). However, Pearson et al. have demonstrated participants can possess a good

404 metacognitive understanding of their own imagery experience, with subjective ratings of image

405 vividness being highly predictive of subsequent impact of imagery on conscious perceptual experience

406 (Pearson, Rademaker, \& Tong, 2011). Brain imaging studies have also linked differences in the

407 introspective experience of mental imagery to differential cortical activation (Cui, Jeter, Yang,

408 Montague, \& Eagleman, 2007; Slotnick, Thompson, \& Kosslyn, 2012). Second, imagery tasks are

409 known to recruit a diverse array of cognitive components including executive functions (Logie, 1995,

410 2003; Pearson, et al., 1996; Rudkin et al, 2007). Consequently it is possible that the relationship of

411 VVIQ performance and $\mathrm{BD}$ is due to some executive factor unrelated to the visual nature of the

412 imagery concerned. The novel finding of an independent, consistent and statistically reliable

413 relationship between VPT performance and BD clarifies the issue: a purer measure of visual memory is

414 reliably related to body dissatisfaction: again this pattern is consistent with the idea that strong visual

415 representative abilities can protect against body dissatisfaction. 
Although superficially it may seem that VVIQ and VPT may index similar functions - one

417 would expect strong visual representational abilities to correlate with vividness of visual imagery - in

418 fact the correlation in our sample between VPT and VVIQ is small and non-significant $(r(n=110)=$ -

$419.11, \mathrm{p}=.254)$. Bull, Pearson and Hamilton (2007) assessed correlations between VPT and VVIQ as

420 part of a larger-scale study, and reported a slightly higher correlation between VPT and VVIQ $(r=.22$,

$421 \mathrm{p}<.05)$ : they also noted in exploratory factor analysis that VVIQ loaded more on a measure of general

422 executive functioning whilst VPT loaded on a measure of visual representation. On these bases, it

423 seems reasonable to speculate that differences in the profiles of body dissatisfaction relationships

424 between VVIQ and VPT may well centre on the fact that VVIQ has a more substantive executive

425 component, broadly consistent with our findings that VPT appears to be a stronger predictor of VABD

426 (a visual task) than it was the questionnaire-based BSQ.

Stroop interference correlated with every variable in the study apart from global precedence,

428 highlighting its role as a measure of a general inhibitory mechanism. Some of these relationships were

429 very high. The strong and sizeable correlation with age is of little surprise (Comalli, Wapner \&

430 Werner, 1962). A strong relationship with BMI is also consistent with previous literature (e.g.

431 Fagundo, et al., 2012). In the context of the regression models, Stroop was a moderate predictor of

432 BSQ but, was not a significant predictor of VABD. Looking at the high correlations between Stroop

433 interference, BMI, VABD and BSQ it is plausible to explain this by assuming that the relationship

434 between Stroop interference and body dissatisfaction is heavily driven by the links between obesity and

435 inhibition: VABD is more heavily influenced by actual obesity than BSQ (given that the participant has

436 to select a representation of their current body state) and hence the relationship to Stroop may have

437 been substantially reduced in the regression models because they controlled explicitly for BMI, and

438 more attenuated in VABD prediction rather than BSQ prediction. Given this it seems reasonable to

439 hypothesize separable relationships between visual short-term memory and body dissatisfaction and 
440 between executive functions and body dissatisfaction, a testable hypothesis that should be taken up in

441 future research. One obvious possible explanation is that increased inhibitory control may enable

442 people to inhibit unwanted negative body-related thoughts, thus mediating dissatisfaction.

443 It is necessary to briefly consider those functions that were not apparently related to either

444 VABD or BSQ, with due deference to the axiomatic absence of evidence not representing evidence of

445 absence. We failed to observe any relationship between spatial sequence memory and BD, nor between

446 global and local processing and BD in our sample. Hence, whilst acknowledging that these are null

447 findings, there is no evidence that the 'normative discontent' (Rodin, et al., 1984) that is seen in the 448 general population is related to intrinsic biases in processing orientation, or to individual variations in

449 spatial sequence memory. As far as we are aware this is the first study attempting to link BD with 450 spatial memory and global precedence.

This study restricted its focus to female participants. Hence we must add the caveat that these

452 data do not necessarily generalize to males, and that research on the relationship between visual 453 cognition and BD in males would be warranted in future. Of particular interest here may be the 454 relatively complex patterns of gender difference in visual and spatial memory ability (e.g. Voyer, 455 Postma, Brake \& Imperato-McGinley, 2007; Cattaneo, Postma \& Vecchi, 2006), and it would be 456 worthwhile to tease out relationships between BD, gender and visual cognition in future. In the light of 457 these gender differences, it must be made explicit here that the current data do not support the 458 argument that females are more prone to BD or eating disorders as a consequence of gender differences 459 in visual processing. Such a claim would be groundless, and go way beyond our current claim which is 460 to say that we have observed evidence consistent with a role for visual processing in establishing, 461 maintaining or protecting against BD.

The Working Memory model (Baddeley, 2000; Baddeley et al, 2011) was advanced earlier as a 463 potentially useful framework for understanding the role of visual imagery in relation to BD. Given the 
464 current data, it seems likely that there is a substantive relationship between two components of working 465 memory and BD. Efficient functioning in both the visuo-spatial sketch pad (VSSP) and the central 466 executive (CE) seems to be associated with lower BD. The executive processing relationship was 467 stronger for BSQ than for VABD, leading to the tentative conclusion that the BSQ, perhaps by virtue 468 of its use of a verbal questionnaire format, may have more executive demands. Nonetheless, an 469 independent and reliable relationship between Stroop interference to the VABD demonstrates a 470 relationship between $\mathrm{CE}$ and $\mathrm{BD}$ that extends beyond the nature of the stimulus materials and 471 implicates the $\mathrm{CE}$ in $\mathrm{BD}$. Meanwhile, a relatively small but significant independent contribution of the 472 VPT in predicting both BSQ and VABD demonstrates the importance of visual STM to sustaining BD. The present study allows a more detailed understanding of the relationship between the VSSP and BD, because only visual STM predicted BD: spatial STM had no such association. There is a reasonable consensus that the VSSP is likely to be divisible into a primarily visual and a primarily spatial system (e.g. Della Sala, et al., 1999; Logie, 1995) though there has been debate about the precise nature of these systems and how they interact (e.g. Logie, 2011; Darling, Della Sala \& Logie, 2009). There is also evidence that visual and spatial imagery recruit different cognitive systems (Quinn \& Ralston, 1986; McConnell \& Quinn, 2000). With these patterns in mind, the present results suggest that the systems associated with $\mathrm{BD}$ are visual, rather than spatial, in nature: there was no evidence at 481 all for a link between spatial sequential memory (on the Corsi task) and BD. a new component known as the episodic buffer (EB), with a role in combining information held in separate stores: the impact of this structure is less clear, and remains to be probed in future research and theorizing. The inhibition and attentional demands of the Stroop task used in the current research are more related to the Central Executive than they are clearly linked to the episodic buffer. As a 
488 simultaneously held in different STM and LTM stores, the EB might well be involved in sustaining 489 distorted body-image related representations; for example when an individual may be thinking about 490 their appearance it may provide access to episode-specific information which could easily be a source 491 of biasing or negatively-valenced information. Given the role of the EB role in holding together (or 492 'binding') information held in parallel information streams in order to carry out particular task 493 demands (e.g. Darling, Allen, Havelka, Campbell \& Rattray, 2011, Allen, Havelka, Falcon, Evans \& 494 Darling, 2014), it may be the case that the efficiency of the EB may, speculatively, influence the 495 robustness of self-image representations. However, this is speculation in extremis, and a fuller understanding awaits a fuller understanding of the role of the EB in imagery tasks in general, an

497 enterprise that has not really been pursued at the present time (Pearson, 2006). We should also note that 498 the working memory model is not the only notable model of mental imagery that separates visual and 499 spatial processes; this explanation is also largely consistent with other approaches to imagery (Farah, 500 Hammond, Levine \& Calvanio, 1988, Kozhevnikov, Kosslyn \& Shephard, 2005). In summary, the results of the current study indicate that some measures of visuospatial 502 cognitive function are predictive of body image measures. In order to understand the processes underlying body dissatisfaction, researchers would therefore be well advised to consider visual imagery and visual memory features, and we argue that the well-established working memory model proposes constructs that may help advance this understanding. It is possible that a vivid and veridical visual

506 imagery system offers a degree of immunity to the various insults to body image that are synonymous 507 with life in the modern world, perhaps by providing a robust parallel representation of the body that is 508 either less or differentially affected by messages in the media and society. How this representation is 509 established and maintained is a question for future research. At present we have identified a role for 510 visual STM and the CE but other aspects of cognition may well be involved. As a final note, we wish 511 to make it clear that we completely acknowledge the massive importance of social and media 
512 representations to endemic, normative $\mathrm{BD}$, and would not seek to underplay this. However, the finding

513 that visual representation is a crucial mediator of $\mathrm{BD}$ is also of key importance, and we hope that this

514 finding can be the basis for development of innovative approaches to BD that may help mitigate the

515 pervasive and debilitating social effects of this strikingly prevalent phenomenon.

516 
518 The authors would like to acknowledge the invaluable assistance of XXXXXXX, XXXXXX

519 and XXXXXX in collecting the data reported in this study. [Names deleted to preserve anonymity, will 520 provide if MS accepted]

\section{Data Archive}


528

529

530 Abbate-Daga, G., Gramaglia, C., Amianto, F., Marzola, E., \& Fassino, S. (2010). Attachment

\section{References} And Mental Disease, 198(7), 520-524. doi:10.1097/nmd.0b013e3181e4c6f7

Allen, R.J, Havelka, J., Falcon, T., Evans, S., \& Darling, S. (2014). Modality Specificity and Integration in Working Memory: Insights From Visuospatial Bootstrapping. Journal Of Experimental Psychology: Learning, Memory, And Cognition. doi:10.1037/xlm0000058

Andrade, J., Kavanagh, D., \& Baddeley, A. (1997). Eye-movements and visual imagery: A working memory approach to the treatment of post-traumatic stress disorder. British Journal Of Clinical Psychology, 36(2), 209-223. doi:10.1111/j.2044-8260.1997.tb01408.x

Aspen, V., Darcy, A., \& Lock, J. (2013). A review of attention biases in women with eating disorders. Cognition \& Emotion, 27(5), 820-838. doi:10.1080/02699931.2012.749777

Attie, I., \& Brooks-Gunn, J. (1989). Development of eating problems in adolescent girls: A longitudinal study. Developmental Psychology, 25(1), 70-79. doi:10.1037//0012-1649.25.1.70

Auchus, M., Kose, G., \& Allen, R. (1993). Body-image distortion and mental imagery. Perceptual And Motor Skills, 77(3), 719-728. doi:10.2466/pms.1993.77.3.719

Baddeley, A.D (1996). Exploring the Central Executive. The Quarterly Journal Of Experimental Psychology A, 49(1), 5-28. doi:10.1080/027249896392784 
547 Baddeley, A.D. (2000). The episodic buffer: a new component of working memory?. Trends In $548 \quad$ Cognitive Sciences, 4(11), 417-423. doi:10.1016/s1364-6613(00)01538-2

549 Baddeley, A.D., \& Andrade, J. (2000). Working memory and the vividness of imagery. Journal of Experimental Psychology: General, 129(1), 126-145. doi: 10.1037//0096-3445.129.1.126

551 Baddeley, A.D., \& Hitch, G.J. (1974). Working Memory . Academic Press, 8, 47-89.

552 Baddeley, A.D., Allen, R.J., \& Hitch, G.J. (2011). Binding in visual working memory: The role of the 553 episodic buffer. Neuropsychologia, 49(6), 1393-1400.

554 doi:10.1016/j.neuropsychologia.2010.12.042

555 Baker, J., Williamson, D., \& Sylve, C. (1995). Body image disturbance, memory bias, and body 556 dysphoria: Effects of negative mood induction. Behavior Therapy, 26(4), 747-759.

557 doi:10.1016/s0005-7894(05)80043-7

558 Boeka, A., \& Lokken, K. (2008). Neuropsychological performance of a clinical sample of extremely 559 obese individuals. Archives Of Clinical Neuropsychology, 23(4), 467-474.

$560 \quad$ doi:10.1016/j.acn.2008.03.003

561 Bulik, C., Wade, T., Heath, A., Martin, N., Stunkard, A., \& Eaves, L. (2001). Relating body mass 562 index to figural stimuli: population-based normative data for Caucasians. Int J Obes Relat Metab $563 \quad$ Disord, 25(10), 1517-1524. doi:10.1038/sj.ijo.0801742

564 Bull, R., Pearson, D., \& Hamilton, C. (2007). Measurement and Interference of Visual-Spatial 565 Memory: Full Research Report ESRC End of Award Report, RES-000-22-1507. Swindon: ESRC. 566 Retrieved from http://www.esrc.ac.uk/my-esrc/grants/RES-000-22-1507/read 
567 Cattaneo, Z., Postma, A., \& Vecchi, T. (2006). Gender differences in memory for object and word $568 \quad$ locations. The Quarterly Journal Of Experimental Psychology, 59(05), 904-919.

569 doi:10.1080/02724980543000079

570 Comalli, P., Wapner, S., \& Werner, H. (1962). Interference Effects of Stroop Color-Word Test in

571 Childhood, Adulthood, and Aging. The Journal Of Genetic Psychology, 100(1), 47-53.

572 doi:10.1080/00221325.1962.10533572

573 Cserjési, R., Luminet, O., Poncelet, A., \& Lénárd, L. (2009). Altered executive function in obesity.

574 Exploration of the role of affective states on cognitive abilities. Appetite, 52(2), 535-539.

575 doi:10.1016/j.appet.2009.01.003

576 Cserjési, R., Molnar, D., Luminet, O., \& Lénárd, L. (2007). Is there any relationship between obesity 577 and mental flexibility in children? Appetite, 49(3), 675-678. doi:10.1016/j.appet.2007.04.001

578 Cocude, M., Charlot, V., \& Denis, M. (1997). Latency and duration of visual mental images in normal 579 and depressed subjects. Journal Of Mental Imagery, 21(1-2), 127-142

580 Cooper, P., Taylor, M., Cooper, Z., \& Fairburn, C. (1987). The development and validation of the body 581 shape questionnaire. International Journal Of Eating Disorders, 6(4), 485-494.

582 doi:10.1002/1098-108x(198707)6:4<485::aid-eat2260060405>3.0.co;2-o

583 Corsi, P. (1972). Human Memory and the Medial Temporal Region of the Brain (Ph.D.). McGill $584 \quad$ University. 
585 Cui, X., Jeter, C., Yang, D., Montague, P., \& Eagleman, D. (2007). Vividness of mental imagery:

586 Individual variability can be measured objectively. Vision Research, 47(4), 474-478.

587 doi:10.1016/j.visres.2006.11.013

588 Darling, S., Allen, R.J, Havelka, J., Campbell, A., \& Rattray, E. (2012). Visuospatial bootstrapping:

589 Long-term memory representations are necessary for implicit binding of verbal and visuospatial 590 working memory. Psychon Bull Rev, 19(2), 258-263. doi:10.3758/s13423-011-0197-3

591 Darling, S., Martin, D., Hellmann, J., \& Memon, A. (2009). Some witnesses are better than others. 592 Personality And Individual Differences, 47(4), 369-373. doi:10.1016/j.paid.2009.04.010

593 Darling, S., Della Sala, S., \& Logie, R. (2009). Dissociation between appearance and location within 594 595 visuo-spatial working memory. The Quarterly Journal Of Experimental Psychology, 62(3), 417425. doi:10.1080/17470210802321984

596 597

598 599 600

601 602 603 604

Della Sala, S., Gray, C., Baddeley, A., \& Wilson, L. (1997). The visual patterns test: A test of shortterm visual recall. Bury St, Edmunds: Thames Valley Test Company.

Della Sala, S., Gray, C., Baddeley, A., Allamano, N., \& Wilson, L. (1999). Pattern span: a tool for unwelding visuo-spatial memory. Neuropsychologia, 37(10), 1189-1199. doi:10.1016/s00283932(98)00159-6

Drewnowski, A., \& Yee, D. (1987). Men and body image: are males satisfied with their body weight?. Psychosomatic Medicine, 49(6), 626-634. doi:10.1097/00006842-198711000-00008

Dobson, K., \& Dozois, D. (2004). Attentional biases in eating disorders: A meta-analytic review of Stroop performance. Clinical Psychology Review, 23(8), 1001-1022. 
doi:10.1016/j.cpr.2003.09.004

606 Evans, C., \& Dolan, B. (1993). Body shape questionnaire: Derivation of shortened "alternate forms". 607 International Journal Of Eating Disorders, 13(3), 315-321. doi:10.1002/1098-

608 $108 \times(199304) 13: 3<315:$ :aid-eat2260130310>3.0.co;2-3

609 Fagundo, A., de la Torre, R., Jiménez-Murcia, S., Agüera, Z., Granero, R., Tárrega, S., Botella, C.,

610 Baños, R., Fernández-Real, J.M., Rodríguez, R., Forcano, L., Frühbeck, G., Gómez-Ambrosi, J., 611 Tinahones, F.J., Fernández-García, J.C., Casanueva, F.F. \& Fernández-Aranda, F.F. (2012). 612 Executive Functions Profile in Extreme Eating/Weight Conditions: From Anorexia Nervosa to 613 Obesity. Plos ONE, 7(8), e43382. doi:10.1371/journal.pone.0043382

614 Fairburn, C., Shafran, R., \& Cooper, Z. (1999). A cognitive behavioural theory of anorexia nervosa. 615 Behaviour Research And Therapy, 37(1), 1-13. doi:10.1016/s0005-7967(98)00102-8

616 Farah, M., Hammond, K., Levine, D., \& Calvanio, R. (1988). Visual and spatial mental imagery:

617 Dissociable systems of representation. Cognitive Psychology, 20(4), 439-462. doi:10.1016/0010$618 \quad 0285(88) 90012-6$

619 Fazio, R., Jackson, J., Dunton, B., \& Williams, C. (1995). Variability in automatic activation as an 620 unobstrusive measure of racial attitudes: A bona fide pipeline?. Journal Of Personality And $621 \quad$ Social Psychology, 69(6), 1013-1027. doi:10.1037//0022-3514.69.6.1013

622 Feusner, J., Moller, H., Altstein, L., Sugar, C., Bookheimer, S., Yoon, J., \& Hembacher, E. (2010). 623 Inverted face processing in body dysmorphic disorder. Journal Of Psychiatric Research, 44(15), 624 1088-1094. doi:10.1016/j.jpsychires.2010.03.015 
625 Freeman, R., Touyz, S., Sara, G., Rennie, C., Gordon, E., \& Beumont, P. (1991). In the eye of the 626 beholder: Processing body shape information in anorexic and bulimic patients. International Journal Of Eating Disorders, 10(6), 709-714. doi:10.1002/1098-108x(199111)10:6<709::aideat2260100609>3.0.co; $2-n$

629 Furnham, A., \& Calnan, A. (1998). Eating disturbance, self-esteem, reasons for exercising and body weight dissatisfaction in adolescent males. European Eating Disorders Review, 6(1), 58-72. doi:10.1002/(sici)1099-0968(199803)6:1<58::aid-erv184>3.3.co;2-m

632 Furnham, A., Badmin, N., \& Sneade, I. (2002). Body Image Dissatisfaction: Gender Differences in 633 Eating Attitudes, Self-Esteem, and Reasons for Exercise. The Journal Of Psychology, 136(6), 634 581-596. doi:10.1080/00223980209604820

635 Green, M., \& Rogers, P. (1993). Selective attention to food and body shape words in dieters and 636 637 restrained nondieters. International Journal Of Eating Disorders, 14(4), 515-517. doi:10.1002/1098-108x(199312)14:4<515::aid-eat2260140417>3.0.co;2-e

638 Greenwald, A., Nosek, B., \& Banaji, M. (2003). "Understanding and using the Implicit Association 639 640 Test: I. An improved scoring algorithm". Journal Of Personality And Social Psychology, 85(2), 197-216. doi: 10.1037/0022-3514.85.2.197.

641 Gunstad, J., Paul, R., Cohen, R., Tate, D., Spitznagel, M., \& Gordon, E. (2007). Elevated body mass 642 index is associated with executive dysfunction in otherwise healthy adults. Comprehensive 643 Psychiatry, 48(1), 57-61. doi:10.1016/j.comppsych.2006.05.001 
644 Hargreaves, D., \& Tiggemann, M. (2002). The Effect Of Television Commercials On Mood And Body 645 Dissatisfaction: The Role Of Appearance-Schema Activation. Journal Of Social And Clinical $646 \quad$ Psychology, 21(3), 287-308. doi:10.1521/jscp.21.3.287.22532

647 Holmes, E., James, E., Coode-Bate, T., \& Deeprose, C. (2009). Can Playing the Computer Game 648 "Tetris" Reduce the Build-Up of Flashbacks for Trauma? A Proposal from Cognitive Science. 649 Plos ONE, 4(1), e4153. doi:10.1371/journal.pone.0004153

650 Kemps, E., \& Tiggemann, M. (2013). Hand-held dynamic visual noise reduces naturally occurring 651 food cravings and craving-related consumption. Appetite, 68, 152-157. 652 doi:10.1016/j.appet.2013.05.001

653 Killen, J., Taylor, C., Hayward, C., Haydel, K., Wilson, D., \& Hammer, L., Kraemer, H., Blair654 Greiner, A. \& Strachowski, D. (1996). Weight concerns influence the development of eating 655 disorders: A 4-year prospective study. Journal Of Consulting And Clinical Psychology, 64(5), 656 936-940. doi:10.1037/0022-006x.64.5.936

657 Kozhevnikov, M., Kosslyn, S., \& Shephard, J. (2005). Spatial versus object visualizers: A new 658 characterization of visual cognitive style. Memory \& Cognition, 33(4), 710-726. 659 doi:10.3758/bf03195337

660 Kosslyn, S. (1981). The medium and the message in mental imagery: A theory. Psychological Review, 661 88(1), 46-66. doi:10.1037//0033-295x.88.1.46 
662 Kosslyn, S., Ball, T., \& Reiser, B. (1978). Visual images preserve metric spatial information: Evidence 663 from studies of image scanning. Journal Of Experimental Psychology: Human Perception And 664 Performance, 4(1), 47-60. doi:10.1037//0096-1523.4.1.47

665 Kostanski, M., \& Gullone, E. (1999). Dieting and Body Image in the Child's World: Conceptualization 666 and Behavior. The Journal Of Genetic Psychology, 160(4), 488-499.

667 doi:10.1080/00221329909595561

668 669

670 671 672 673 674 675 676 677 678 679

Lee, M., \& Shafran, R. (2004). Information processing biases in eating disorders. Clinical Psychology Review, 24(2), 215-238. doi:10.1016/j.cpr.2003.10.004

Lena, S., Fiocco, A., \& Leyenaar, J. (2004). The Role of Cognitive Deficits in the Development of Eating Disorders. Neuropsychol Rev, 14(2), 99-113. doi:10.1023/b:nerv.0000028081.40907.de

Logie, R. (1995). Visuo-spatial working memory. Hove, East Sussex: L. Erlbaum Associates.

Logie, R. (2003). Spatial and visual working memory: A mental workspace. In D.E. Irwin \& B.H. Ross (Eds.) Cognitive Vision: The Psychology of Learning and Motivation Volume 42. (pp. 37-78). DOI: $10.1016 / \mathrm{S} 0079-7421(03) 01002-8$

Logie, R. (2011). The Functional Organization and Capacity Limits of Working Memory. Current Directions In Psychological Science, 20(4), 240-245. doi:10.1177/0963721411415340

Lusher, J., Chandler, C., \& Ball, D. (2004). Alcohol dependence and the alcohol Stroop paradigm: evidence and issues. Drug and Alcohol Dependence, 75(3), 225-231. 
680 MacLeod, C. (1991). Half a century of research on the Stroop effect: An integrative review. Psychological Bulletin, 109(2), 163-203. doi:10.1037/0033-2909.109.2.163

682

683

684

685

686

687

688

689

690

691

692

693

694

695

696

697

698

Marks, D. (1973). Visual imagery differences in the recall of pictures. British Journal of Psychology, 64(1), 17-24. doi:10.1111/j.2044-8295.1973.tb01322.x

Martin, D., \& Macrae, C. (2010). Processing style and person recognition: Exploring the face inversion effect. Visual Cognition, 18(2), 161-170. doi:10.1080/13506280902868793

McConnell, J., \& Quinn, J. (2000). Interference in Visual Working Memory. The Quarterly Journal Of Experimental Psychology A, 53(1), 53-67. doi:10.1080/027249800390664

Mciza, Z., Goedecke, J., Steyn, N., Charlton, K., Puoane, T., \& Meltzer, S., Levitt, N.S. \& Lambert, E.V.. (2005). Development and validation of instruments measuring body image and body weight dissatisfaction in South African mothers and their daughters. Public Health Nutrition, 8(05). doi:10.1079/phn2005814

McKelvie, S. (1995). The VVIQ as a psychometric test of individual differences in visual imagery vividness: A critical quantitative review and plea for direction. Journal Of Mental Imagery, 19(34), 1-106.

Milner, B. (1971). Interhemispheric differences in the localization of psychological processes in man. British Medical Bulletin, 27(3), 272-277.

Miyake, A., Friedman, N., Emerson, M., Witzki, A., Howerter, A., \& Wager, T. (2000). The Unity and Diversity of Executive Functions and Their Contributions to Complex "Frontal Lobe" Tasks: A 
699

700

701

702

703

704

705

706

707

708

709

710

711

712

713

714

715

716

717

Latent Variable Analysis . Cognitive Psychology, 41(1), 49-100. Retrieved from http://www.sciencedirect.com/science/article/pii/S001002859990734X

Navon, D. (1977). Forest before trees: The precedence of global features in visual perception. Cognitive Psychology, 9(3), 353-383. doi:10.1016/0010-0285(77)90012-3

Nederkoorn, C., Braet, C., Van Eijs, Y., Tanghe, A., \& Jansen, A. (2006). Why obese children cannot resist food: The role of impulsivity. Eating Behaviors, 7(4), 315-322. doi:10.1016/j.eatbeh.2005.11.005

Nederkoorn, C., Smulders, F., Havermans, R., Roefs, A., \& Jansen, A. (2006). Impulsivity in obese women. Appetite, 47(2), 253-256. doi:10.1016/j.appet.2006.05.008

Oertel, V., Rotarska-Jagiela, A., van de Ven, V., Haenschel, C., Grube, M., \& Stangier, U., Maurer, K. \& Linden, D.E. (2009). Mental imagery vividness as a trait marker across the schizophrenia spectrum. Psychiatry Research, 167(1-2), 1-11. doi:10.1016/j.psychres.2007.12.008

Ohring, R., Graber, J., \& Brooks-Gunn, J. (2002). Girls' recurrent and concurrent body dissatisfaction: Correlates and consequences over 8 years. International Journal Of Eating Disorders, 31(4), 404-415. doi:10.1002/eat.10049

Paivio, A. (1991). Dual coding theory: Retrospect and current status. Canadian Journal Of Psychology/Revue Canadienne De Psychologie, 45(3), 255-287. doi:10.1037/h0084295

Paxton, S., Neumark-Sztainer, D., Hannan, P., \& Eisenberg, M. (2006). Body Dissatisfaction Prospectively Predicts Depressive Mood and Low Self-Esteem in Adolescent Girls and Boys. 
718

719

720

721

722

723

724

725

726

727

728

729

730

731 732

733 734

735

736

Journal Of Clinical Child \& Adolescent Psychology, 35(4), 539-549. doi:10.1207/s15374424jccp3504_5

Pearson, D.G. (2006). The episodic buffer: Implications and connections with visuo-spatial research. In: T. Vecchi (Ed.), Imagery and Spatial Cognition: Methods, Models and Cognitive Assessment (pp.139-153). Amsterdam: John Benjamins Publishing Company.

Pearson, D., \& Logie, R. (2004). Effects of stimulus modality and working memory load on mental synthesis performance. Imagination, Cognition And Personality, 23(2), 183-191. doi:10.2190/krqb-0ced-nx6j-hq72

Pearson, D., Deeprose, C., Wallace-Hadrill, S., Heyes, S., \& Holmes, E. (2013). Assessing mental imagery in clinical psychology: A review of imagery measures and a guiding framework. Clinical Psychology Review, 33(1), 1-23. doi:10.1016/j.cpr.2012.09.001

Pearson, D., Logie, R., \& Green, C. (1996). Mental manipulation, visual working memory, and executive processes. Psychologische Beitrage, 38(3-4), 324-342.

Pearson, J., Rademaker, R., \& Tong, F. (2011). Evaluating the Mind's Eye: The Metacognition of Visual Imagery. Psychological Science, 22(12), 1535-1542. doi:10.1177/0956797611417134

Perpiñá, C., Hemsley, D., Treasure, J., \& De Silva, P. (1993). Is the selective information processing of food and body words specific to patients with eating disorders?. International Journal Of Eating Disorders, 14(3), 359-366. doi:10.1002/1098-108x(199311)14:3<359::aideat2260140314>3.0.co;2-g 
737 Presnell, K., Bearman, S., \& Stice, E. (2004). Risk factors for body dissatisfaction in adolescent boys

738 and girls: A prospective study. International Journal Of Eating Disorders, 36(4), 389-401.

739 doi:10.1002/eat.20045

740 Pylyshyn, Z. (1981). The imagery debate: Analogue media versus tacit knowledge. Psychological

$741 \quad$ Review, 88(1), 16-45. doi:10.1037//0033-295x.88.1.16

742 Quinn, J. (1996). Irrelevant Pictures in Visual Working Memory. The Quarterly Journal of

743 Experimental Psychology Section A, 49(1), 200-215. doi:10.1080/713755613

744 Quinn, J., \& McConnell, J. (2006). The interval for interference in conscious visual imagery. Memory, 14(2), 241-252. doi:10.1080/09658210500210019

746 Quinn, J., \& Ralston, G. (1986). Movement and attention in visual working memory. The Quarterly

747 Journal Of Experimental Psychology Section A, 38(4), 689-703.

748 doi:10.1080/14640748608401621

749 Roberts, M., Tchanturia, K., Stahl, D., Southgate, L., \& Treasure, J. (2007). A systematic review and

750 meta-analysis of set-shifting ability in eating disorders. Psychological Medicine, 37(08), 1075.

$751 \quad$ doi: $10.1017 / \mathrm{s} 0033291707009877$

752 Rodin, J., Silberstein, L., \& Striegel-Moore, R. (1984). Women and weight: A normative discontent.

753 Nebraska Symposium On Motivation, 32, 267-307.

754 Rudkin, S., Pearson, D., \& Logie, R. (2007). Executive processes in visual and spatial working

755 memory tasks. The Quarterly Journal Of Experimental Psychology, 60(1), 79-100.

756 doi:10.1080/17470210600587976 
757 Slotnick, S., Thompson, W., \& Kosslyn, S. (2012). Visual memory and visual mental imagery recruit 758 common control and sensory regions of the brain. Cognitive Neuroscience, 3(1), 14-20. doi:10.1080/17588928.2011.578210

760 Smeets, M., Klugkist, I., Rooden, S., Anema, H., \& Postma, A. (2009). Mental body distance

761

762

763

764

765

766

767

768

769

770

771

772

773

774 comparison: A tool for assessing clinical disturbances in visual body image. Acta Psychologica, 132(2), 157-165. doi:10.1016/j.actpsy.2009.03.011

Stice, E., \& Whitenton, K. (2002). Risk factors for body dissatisfaction in adolescent girls: A longitudinal investigation. Developmental Psychology, 38(5), 669-678. doi:10.1037//00121649.38.5.669

Stice, E., Hayward, C., Cameron, R., Killen, J., \& Taylor, C. (2000). Body-image and eating disturbances predict onset of depression among female adolescents: A longitudinal study. Journal Of Abnormal Psychology, 109(3), 438-444. doi:10.1037//0021-843x.109.3.438

Stroop, J. (1935). Studies of interference in serial verbal reactions. Journal Of Experimental Psychology, 18(6), 643-662. doi:10.1037/h0054651

Stunkard, A., Sørensen, T., Hanis, C., Teasdale, T., Chakraborty, R., Schull, W., \& Schulsinger, F. (1986). An Adoption Study of Human Obesity. New England Journal Of Medicine, 314(4), 193198. doi:10.1056/nejm198601233140401

Thompson, J. (1999). Exacting beauty. Washington, DC: American Psychological Association. 
775 Tiggemann, M. (2006). The Role of Media Exposure in Adolescent Girls' Body Dissatisfaction and 776 Drive for Thinness: Prospective Results. Journal Of Social And Clinical Psychology, 25(5), 523541. doi:10.1521/jscp.2006.25.5.523

778 Vitousek, K., \& Hollon, S. (1990). The investigation of schematic content and processing in eating 779 disorders. Cognitive Therapy And Research, 14(2), 191-214. doi:10.1007/bf01176209

780 Voyer, D., Postma, A., Brake, B., \& Imperato-McGinley, J. (2007). Gender differences in object 781 location memory: A meta-analysis. Psychonomic Bulletin \& Review, 14(1), 23-38. 782 doi:10.3758/bf03194024

783 Williams, J. M. G., Mathews, A., \& MacLeod, C. (1996). The emotional Stroop task and 784 psychopathology. Psychological bulletin, 120(1), 3. 


\section{Table $\mathbf{1}_{\text {(on next page) }}$}

Summary statistics of principal variables

Key to abbreviations: BMI $=$ Body Mass Index, $\mathrm{BSQ}=$ Body ShapeQuestionnaire score, VABD $=$ Visual-Analogue Body Dissatisfaction differencescore, $\mathrm{VVIQ}=$ Vividness of Visual Imagery Questionnaire score, VPT =computerized Visual Patterns Task span, Corsi = computerized Corsi blocks taskspan, GP = computerized Global Precedence score, $\mathrm{SI}=$ Stroop interferencescore. 


\begin{tabular}{ccccccc}
\hline Variable & $\begin{array}{c}\text { Valid } N \\
\text { observations }\end{array}$ & Mean & $S D$ & Min & Max & Skewness \\
& & & & & & \\
\hline BMI & 110 & 24.25 & 4.53 & 16.85 & 39.76 & 1.42 \\
Age & 110 & 26.53 & 10.20 & 18 & 62 & 1.63 \\
BSQ & 110 & 45.18 & 17.90 & 16 & 89 & 0.32 \\
VABD & 110 & 1.34 & 1.23 & -1.00 & 6.00 & 0.99 \\
VVIQ & 110 & 75.86 & 20.86 & 42.00 & 145.00 & 0.49 \\
VPT & 110 & 9.93 & 2.85 & 4 & 15 & -0.22 \\
Corsi & 110 & 6.26 & 1.34 & 4 & 9 & -0.24 \\
GP & 108 & -65.91 & 267.73 & -930 & 758 & -0.54 \\
SI & 110 & 6.12 & 3.79 & -0.75 & 19.50 & 0.95 \\
\hline
\end{tabular}




\section{Table 2 (on next page)}

Correlations between variables

This table shows the Pearson correlation coefficients between thedifferent variables measured in the sample. Key to abbreviations: ${ }^{*}=\mathrm{p}<.05$; ** $=\mathrm{p}<.01$; *** $=\mathrm{p}<.001$; BMI = Body Mass Index, BSQ = BodyShape Questionnaire score, VABD = Visual-Analogue Body Dissatisfactiondifference score, VVIQ = Vividness of Visual Imagery Questionnaire score, VPT =computerized Visual Patterns Task span, Corsi = computerized Corsi blocks taskspan, GP = computerized Global Precedence score, $\mathrm{SI}=$ Stroop interferencescore. 
PeerJ Reviewing Manuscript

\begin{tabular}{|c|c|c|c|c|c|c|c|c|}
\hline & Age & BSQ & VABD & VVIQ & VPT & Corsi & GP & SI \\
\hline$N$ & 108 & 108 & 108 & 108 & 108 & 108 & 106 & 108 \\
\hline BMI & $.33 * * *$ & .03 & $.53 * * *$ & .12 & -.07 & -.07 & -.06 & $.36^{* * *}$ \\
\hline Age & & -.16 & .15 & .03 & -.11 & $-.22 *$ & $-.20 *$ & $.45 * * *$ \\
\hline BSQ & & & $.56^{* * *}$ & $.41 * * *$ & $-.22 *$ & -.03 & .06 & $.23 *$ \\
\hline VABD & & & & $.34 * * *$ & $-.31 * *$ & -.14 & .02 & $.42 * * *$ \\
\hline VVIQ & & & & & -.11 & .03 & -.11 & $.28 * *$ \\
\hline VPT & & & & & & $.50 * * *$ & -.01 & $-.32 * *$ \\
\hline Corsi & & & & & & & .09 & $-.24 *$ \\
\hline GP & & & & & & & & .06 \\
\hline
\end{tabular}




\section{Table 3 (on next page)}

Summaries of the regression models predictingbody dissatisfaction

This table summarizes the models generated in the regression procedures. Two regression models are reported, one predicting scores on the body shape questionnaireand one predicting scores on the visual analogue body dissatisfaction measure.Both analyses employed a two-step method, with age and BMI being enteredinitially as covariates and then the other measures entering in a stepwiseprocedure. The bottom-most model in each analysis in the table is the finalmodel obtained. Key to abbreviations: BMI = Body Mass Index, BSQ = Body ShapeQuestionnaire score, VABD = Visual-Analogue Body Dissatisfaction differencescore, $\mathrm{VVIQ}=$ Vividness of Visual Imagery Questionnaire score, VPT =computerized Visual Patterns Task span, Corsi = computerized Corsi blocks taskspan, GP = computerized Global Precedence score, $\mathrm{SI}=$ Stroop interference score, $\Delta R^{2}=$ change in $\mathrm{R}^{2}$, lowercase $b$ = regression coefficient, $\beta=$ standardised regression coefficient. Analysis $N$ varies due to exclusion ofparticipants based on Cook's distance. 


\begin{tabular}{|c|c|c|c|c|c|c|}
\hline $\begin{array}{l}\text { Predicted } \\
\text { Outcome }\end{array}$ & Model & Predictor & $\bar{b}$ & $S E b$ & $\beta$ & $\begin{array}{l}\text { Independent } \\
\text { Contribution } \\
\text { to } R^{2+}\end{array}$ \\
\hline$\overline{B S Q}$ & 1 & (Constant) & 39.25 & 9.76 & & \\
\hline \multirow{17}{*}{$\begin{array}{c}\text { Analysis } \\
N=99\end{array}$} & \multirow[t]{2}{*}{$\Delta R^{2}=.04$} & BMI & .59 & .43 & .15 & .01 \\
\hline & & Age & -.38 & .20 & -.20 & .03 \\
\hline & 2 & (Constant) & 12.67 & 9.56 & & \\
\hline & \multirow[t]{3}{*}{$\Delta R^{2}=.25^{* * *}$} & BMI & .39 & .37 & .10 & .01 \\
\hline & & Age & -.34 & .18 & -.18 & .03 \\
\hline & & VVIQ & . 40 & .07 & $.51 * * *$ & .25 \\
\hline & 3 & (Constant) & 18.18 & 9.25 & & \\
\hline & \multirow[t]{4}{*}{$\Delta R^{2}=.07 * *$} & BMI & .22 & .35 & .06 & $<.01$ \\
\hline & & Age & -.57 & .18 & $-.30 * *$ & .07 \\
\hline & & VVIQ & .34 & .07 & $.43 * * *$ & .18 \\
\hline & & SI & 1.51 & .46 & $.31 * *$ & .07 \\
\hline & 4 & (Constant) & 30.38 & 10.88 & & \\
\hline & \multirow{5}{*}{$\Delta R^{2}=.03 *$} & BMI & .25 & .35 & .06 & $<.01$ \\
\hline & & Age & -.57 & .18 & $-.30 * *$ & .068 \\
\hline & & VVIQ & .34 & .07 & $.43 * * *$ & .17 \\
\hline & & SI & 1.17 & .48 & $.24 *$ & .04 \\
\hline & & VPT & -1.05 & .51 & $-.18^{*}$ & .03 \\
\hline VABD & 1 & (Constant) & -1.21 & .52 & & \\
\hline \multirow{11}{*}{$\begin{array}{l}\text { Analysis } \\
N=103\end{array}$} & \multirow[t]{2}{*}{$\Delta R^{2}=.18^{* * *}$} & BMI & .10 & .02 & $.44 * * *$ & .17 \\
\hline & & Age & .00 & .01 & -.03 & $<.01$ \\
\hline & \multirow{4}{*}{$\Delta R^{2}=.11^{2 * *}$} & (Constant) & -2.21 & .55 & & \\
\hline & & BMI & .09 & .02 & $.40 * * *$ & .14 \\
\hline & & Age & .00 & .01 & -.03 & $<.01$ \\
\hline & & VVIQ & .02 & .00 & $.34 * * *$ & .11 \\
\hline & \multirow{5}{*}{$\begin{array}{c}3 \\
\Delta R^{2}=.07 * *\end{array}$} & (Constant) & -1.16 & .61 & & \\
\hline & & BMI & .09 & .02 & $.40 * * *$ & .15 \\
\hline & & Age & .00 & .01 & -.05 & $<.01$ \\
\hline & & VVIQ & .01 & .00 & $.32 * * *$ & .10 \\
\hline & & VPT & -.09 & .01 & $-.03 * *$ & .11 \\
\hline
\end{tabular}

